\title{
$\mathbb{A}$ Economics Bulletin
}

\section{Volume 41, Issue 2}

\section{Competitive effects of horizontal mergers with asymmetric firms}

\author{
Cuong Hung Vuong \\ Edmond Baranes \\ MRE and LabEx Entreprendre, University of Montpellier MRE and LabEx Entreprendre, University of Montpellier
}

\begin{abstract}
This paper aims at investigating the impacts of introducing cost asymmetry in horizontal merger analysis. In the absence of efficiency gains, previous literature states the negative competitive effects of a merger between symmetric firms. We go beyond the literature and show that the result is only likely to hold for a low level of asymmetry. In particular, we build a tractable model with three firms in which one of them has a different cost structure. After merging two symmetrical firms, the outsider always reduces (increases) price (investments), while the insiders choose the opposite strategies. In particular, if the outsider's cost is sufficiently low, the increase in its investment could outweigh the decreases in those of the merged entity, leading to higher total investments post-merger. Similarly, consumer surplus could be improved thanks to the decrease in the outsider's price.
\end{abstract}

Citation: Cuong Hung Vuong and Edmond Baranes, (2021) "Competitive effects of horizontal mergers with asymmetric firms", Economics Bulletin, Vol. 41 No. 2 pp. 734-740.

Contact: Cuong Hung Vuong - HUNG-cuong.vuong@umontpellier.fr, Edmond Baranes - edmond.baranes@umontpellier.fr.

Submitted: January 15, 2021. Published: April 09, 2021. 


\section{Introduction}

The competitive effects of a horizontal merger have been increasingly discussed in Europ $\AA^{1}$. Economists have correctly assessed the impacts by analyzing the behaviors of both insiders or outsiders before and after the merger. One general conclusion is that in the absence of efficiency gains, a merger between two competitors would induce competitive harms for consumers. More precisely, the merger can internalize the externalities by the merging firms leading to higher prices but less innovation. An outsider could respond to the merger by raising its price to match with the increased prices of the insiders due to the effect of strategic price complementarity. On the other hand, the outsider's investment incentive is higher due to the demand expansion effect: investment rewards are greater in a less competitive environment. Thus, if the former effect is stronger, of course, consumers are worse off. Interestingly, if the opposite is true, then the aggregate competitive effects could be still negative if the merger generates no efficiency. The very first and influential model pointing out this result is developed by Motta and Tarantino (2018) (hereinafter $\mathrm{MT})$.

In the fast-moving technology industries such as telecommunications, firms are often asymmetric due to the economics of scale and density, and the so-called first-mover advantages in the liberalization process. Thus, this paper goes beyond the MT's framework and builds a tractable model with triopoly in which one of them can have a different marginal cost. Furthermore, firms could invest in reducing their marginal costs, and thus, ex-post marginal costs depend on both the ex-ante levels and their investments. This paper evaluates the competitive impacts of the merger between the symmetrical firms on the optimal strategies of both inside and outside firms, the total investments, and consumer surplus. We focus on the case in which the pre- and post-merger equilibrium values and the merger's profitability are positive. Our paper abstracts from synergy effects to focus on the roles of the degree of product substitutability and cost asymmetry.

We obtain several interesting results as follows. First, post-merger the insiders would soften competition (higher prices and lower investments) as stressed in MT. The changes in the insiders' behaviors are constrained by the loss of market share, which is linked to the degree of product substitutability and the outsider's response strategy. Second, the merger could yield consumer benefits when comparing the total investments and consumer surplus pre- and post-merger. In particular, the merger has positive competitive effects if the outsider is sufficiently efficient. This is because the outsider is more aggressive after the merger; whereas the insiders, anticipating the outsider's response, have lower incentives to soften competition. In other words, we could evaluate the range of the market parameters in which the merger impacts on both consumer welfare and total investments are positive. Interestingly, this result could occur when all the three firms are symmetric pre-merger and products are highly substitutable.

Related literature. A strand of recent literature assesses the merger impacts on inno-

\footnotetext{
${ }^{1}$ The traditional issues are related to the increases in market concentration or firm market power after a merger. As clearly described in Denicolò and Polo (2019), the debate following the EC's decision on the Dow and Dupont (COMP/M. 7932) case or the Bayer/Monsanto case (COMP/M. 8084) is mostly about the impact on investments in $\mathrm{R} \& \mathrm{D}$ rather than on the traditional issue of the impact of a merger on prices. This consequently has great implications for the high-technology sector (see Calvano and Polo (2020) and Jullien and Lefouili (2018) for comprehensive recent overviews).
} 
vations ${ }^{2}$ As MT, Federico et al. (2017, 2018) also show the negative merger impacts on innovation because the merged firms can reduce duplication in R\&D. Denicolò and Polo (2018 a,b) however argue that the results could be opposite by focusing on analyzing the R\&D expenditures and rewards. In our model, the merger is simply considered as a combination of two firms. Thus, post-merger production and investment cost functions are the same as those pre-merger. Then, we could show that if products are highly substitutable and the outsider firm's cost is relatively low, this would limit the ability of the merged entity to raise the price and decrease investment to avoid an important loss of quantity to the outsider. In other words, the MT's results could hold only when the marginal cost of the outsider is relatively high. Additionally, Bourreau and Jullien (2018) analyze the case of duopolistic firms who can invest in expanding their coverages. In this context, the reward for the firm in a larger area is the monopoly rent. Thus, if the merger is allowed, the single product zone is higher and surpasses the decrease in the multiproduct zone. The authors could prove that both the total coverage and social surplus are higher post-merger. In a more general framework, Bourreau et al. (2019) formally set out different effects of a horizontal merger between duopolist, then tested with various applications. They show conditions under which the merger to monopoly could increase investments, for instance, when firms could invest in raising both product quality and differentiation. Indeed, the situation of merger-to-monopoly could simplify various possible interactions between market players after a merger. Furthermore, in our model, the merged entity provides two differentiated products with the same characteristics as those sold in the market pre-merger. Finally, our paper analyzes the optimal investments for cost-reducing. The results indicate that the total investments could be higher when products are highly substitutable and the outsider firm's cost is efficient.

The structure of our paper is organized as follows. Section 2 lays out the baseline model and characterizes the pre-merger equilibrium. We examine the impacts of the horizontal merger by comparing the pre- and post-merger equilibrium values in Section 3. All the proofs and a numerical case are provided in the Appendix.

\section{The baseline model and pre-merger equilibrium}

\subsection{The model}

We follow the MT's framework with three firms competing à la Bertrand with horizontally differentiated goods. The firms are heterogenous in the marginal costs of production. In particular, we denote the ex-ante marginal cost of firm $i$ by $c_{i}$, where $i=1,2,3$. To simplify our analysis, we assume that only one firm, which is potentially the outsider post-merger, has a different marginal cost. Thus, we have that $c_{1}=c_{2}=c$ and $c_{3}=c-\lambda$ where $\lambda$ represents the asymmetry level. Additionally, $\lambda$ can be positive or negative. In the former (latter) case, firm 3 is considered as being relatively more (less) efficient.

The firms can simultaneously invest in cost-reducing, and thus the ex-post marginal cost of firm $i$ is $\left(c_{i}-\mu_{i}\right)$ where $\mu_{i}$ denotes the investment level of firm $i$. As MT, we assume that the firm $i$ 's cost associated with this investment is independent of the quantity, that is, $\Gamma_{i}=\frac{\mu_{i}^{2}}{2}$. Without loss of generality, we simply assume that other fixed costs are zero.

\footnotetext{
${ }^{2} \mathrm{~A}$ traditional approach to merger impacts on innovations is based on analysis of quantity competition with homogenous goods (see Salant et al., (1983) for the analysis).
} 
On the demand side, we adopt the linear demand model of Singh and Vives (1984). The utility of a representative consumer is

$$
U(q, m)=\sum_{i=1}^{3} q_{i}-\frac{1}{2}\left(\sum_{i=1}^{3} q_{i}^{2}+2 \gamma \sum_{i=1}^{3} \sum_{k>i}^{3} q_{i} q_{k}\right)+m
$$

where $q=\left(q_{1}, q_{2}, q_{3}\right)$ is the vector of quantities, $m$ is the numeraire good, and $\gamma$ measures the degree of substitutability between goods. We aim at analyzing equilibrium with the following properties: (1) Both pre- and post-merger equilibrium values are positive; (2) Ex-post marginal costs are positive; (3) Merger is profitable. Thus, we assume:

Assumption A1: $\gamma<0.478$. This condition is to ensure that the considered merger to be profitable in the absence of efficiency gain ${ }^{3}$, Assumption A2: $\frac{1}{2}<c<1$. Intuitively, the upper bound of $c$ is useful to establish a positive demand in equilibrium, regardless of the investment level. In addition, because firms could invest in reducing the marginal costs, we will want the ex-ante marginal cost to be sufficiently large. Assumption A3: $\lambda<\widehat{\lambda}=\min \left(\lambda_{1}, \lambda_{2}\right)$ where $\lambda_{1}$ and $\lambda_{2}$ are positive $4^{4}$, and depend on the values of $c$ and $\gamma$. Indeed, assumptions $A 2$ and $A 3$ are necessary to ensure the ex-post marginal costs are positive, that is, $c_{i}-\mu_{i}>0$.

From (1), the net utility is simply as the following:

$$
V=U(q, m)-\sum_{i=1}^{3} p_{i} q_{i}
$$

The consumer maximization problem in (2) yields the following demand for firm $i$ :

$$
q_{i}(P)=\frac{-(\gamma+1) p_{i}+\gamma\left(p_{j}+p_{k}\right)}{(1-\gamma)(2 \gamma+1)}+\frac{1}{2 \gamma+1}
$$

where $i \neq j \neq k=1,2,3$ and $P$ is the price vector $\left(p_{1}, p_{2}, p_{3}\right)$. We observe from the demand function specified in (3) that, a firm $i$ 's demand is decreasing in its price, but increasing in the competitors' prices. These impacts are amplified by the degree of substitutability (i.e., $\gamma$ ). This demand function is applied for both pre- and post-merger cases because the merger does not lead to any product elimination in our model.

\subsection{The pre-merger equilibrium}

Each firm chooses simultaneously its price (that is, $p_{i}$ ) and investment (that is, $\mu_{i}$ ) as assumed in the MT's model. In other words, firms choose prices before observing the other firms' investments. In particular, the profit maximization problem of firm $i$ is

$$
\max _{p_{i}, \mu_{i}} \pi_{i}\left(p_{i}, \mu_{i}\right)=\left[p_{i}-\left(c_{i}-\mu_{i}\right)\right] q_{i}(P)-\Gamma_{i}
$$

Assuming for an interior solution, the first-order conditions (FOCs) for the optimal price and investment decisions of firm $i$ are:

$$
\frac{\partial \pi_{i}\left(p_{i}, \mu_{i}\right)}{\partial p_{i}}=q_{i}(p)+\left[p_{i}-\left(c_{i}-\mu_{i}\right)\right] \frac{\partial q_{i}(P)}{\partial p_{i}}=0
$$

\footnotetext{
${ }^{3}$ We will show that insiders will raise prices after the merger. Consequently, if the degree of substitutability is sufficiently low, this does not lead to a significant loss of quantity. Thus, the merger could be profitable in this model.

${ }^{4}$ We show the mathematical formulas and plot $\widehat{a}$ in the Appendix.
} 


$$
\frac{\partial \pi_{i}\left(p_{i}, \mu_{i}\right)}{\partial \mu_{i}}=q_{i}(P)-\frac{\partial \Gamma_{i}}{\partial \mu_{i}}=0
$$

where $\frac{\partial q_{i}(P)}{\partial p_{i}}$ and $\frac{\partial \Gamma_{i}}{\partial \mu_{i}}$ denote the first-order partial derivatives of $q_{i}(P)$ and $\Gamma_{i}$ with respect to $p_{i}$ and $\mu_{i}$, respectively. It can be seen in (6) that the firm $i$ 's marginal benefit of investment is equal to the demand for product $i$. Put it differently, firm $i$ has higher investment incentive if the demand for product $i$ is higher. Then, replacing $q_{i}(P)$ from (3) into (5) and (6), we obtain the best responses of prices and investments:

$$
\begin{gathered}
p_{i}=\frac{c_{i}-\mu_{i}}{2}+\frac{\gamma\left(p_{j}+p_{k}\right)-\gamma+1}{2(\gamma+1)} \\
\mu_{i}=\frac{-(\gamma+1) p_{i}+\gamma\left(p_{j}+p_{k}\right)-\gamma+1}{(1-\gamma)(2 \gamma+1)}
\end{gathered}
$$

There are two remarks regarding the firms' optimal decisions from (7) and (8). Firstly, the firm $i$ 's price is proportional to its ex-post marginal cost, and thus a higher ex-ante marginal cost or lower investment level would lead to a higher price. In turn, the high price reducing the firm's demand implies lower investment incentives. In other words, the firm $i$ 's investment and price are negatively linked. Interestingly, the ex-ante marginal cost $c_{i}$ only appears in (7). This means that, ceteris paribus, firm $i$ would set a higher price, and thus lower investment, for a higher value of $c_{i}$. Secondly, the competitors' prices affect positively both the price and investment of firm $i$. The former effect is the standard result of price strategic complementarity because the firms compete in prices in this paper. The latter effect is emerged from (6): when the competitors' prices are higher, the demand for product $i$ is higher, leading to the higher marginal benefit of the investment.

Solving simultaneously the system indicated in (7) and (8) for three firms, we obtain the equilibrium price and investment levels pre-merger, subscripted with " $E$ ". We summarize the results in the following proposition.

Proposition 1 There exists pre-merger equilibrium under assumptions A1-3. In particular, if firm 3 is relatively more efficient, it would choose a lower price but higher investment relative to those set by the symmetric firms, and vice versa, that is:

$\left\{\begin{array}{l}p_{1}^{E}=p_{2}^{E}>p_{3}^{E} \text { and } \mu_{1}^{E}=\mu_{2}^{E}<\mu_{3}^{E} \text { if } \lambda>0 \\ p_{1}^{E}=p_{2}^{E}<p_{3}^{E} \text { and } \mu_{1}^{E}=\mu_{2}^{E}>\mu_{3}^{E} \text { if } \lambda<0\end{array}\right.$

Proof. See the Appendix.

Proposition 1 states the asymmetric market structure pre-merger when one firm has a different cost. As previously argued, when firm 3 has a relatively lower ex-ante marginal cost, that is, $\lambda>0$, it could relatively set a lower price and thereby capturing a higher market share. Consequently, a higher market share implies that firm 3 would increase its investment, to reduce marginal cost, and so on. Thus, in equilibrium, the price of firm 3 is lower, whereas its investment is higher compared to those of firm 1 and firm 2 . The higher is the value of $\lambda$, the greater are the differences in the prices and investments between the asymmetric firms, that is, firm 3 and firm 1 (or firm 2). The contrasting results occur when $\lambda<0$.

Furthermore, our comparative statics show that if $\lambda>0$, the differences in the asymmetric firms' prices (i.e., $p_{3}^{E}-p_{1}^{E}$ ) and investments (i.e., $\mu_{3}^{E}-\mu_{1}^{E}$ ) are more significant 
for a higher degree of product substitutability (i.e., $\gamma$ ), that is, $\frac{\partial\left(p_{1}^{E}-p_{3}^{E}\right)}{\partial \gamma}>(<) 0$ and $\frac{\partial\left(\mu_{3}^{E}-\mu_{1}^{E}\right)}{\partial \gamma}>(<) 0$ if $\lambda>(<) 0$. Intuitively, when $\gamma$ increases, the firms will want to set lower prices because the products are more comparable from a consumer's viewpoint. This result indicates that if firm 3 is more efficient, its strategy would be more aggressive than that of firm 1 (or firm 2) when products are more substitutable. Next, it is straightforward to compute the total investments, denoted as $T C^{E}$ where:

$$
T C^{E}=\sum_{i=1}^{3} \Gamma_{i}^{E}
$$

By replacing the equilibrium prices and investment into (2), we can compute consumer surplus, denoted as $C S^{E}$. To this end, we can compute the equilibrium firms' profits, and denoted by $\pi_{i}^{E}$ for firm $i$. We can verify that both $C S^{E}$ and $\pi_{i}^{E}$ are positive under the assumptions. In the next section, we will check for the rationality constraint, that is, the profitability of the merger.

\section{The competitive impacts of the merger}

To shed light on the impacts of introducing the asymmetry in horizontal merger analysis, we consider the most simple case in which the symmetric firms are combined to compete against firm 3. Assuming that no efficiency gains are generated post-merger. Post-merger, the merged entity offers two differentiated products. We assume that firms are not able to invest in changing product differentiation, as considered in Bourreau et al. (2019). Thus, the profit function of the merged entity is simply as follows:

$$
\pi_{12}\left(p_{1}, p_{2}, \mu_{1}, \mu_{2}\right)=\left[p_{1}-\left(c_{1}-\mu_{1}\right)\right] q_{1}(p)+\left[p_{2}-\left(c_{2}-\mu_{2}\right)\right] q_{2}(p)-\Gamma_{1}-\Gamma_{2}
$$

The profit function of firm 3 is still given in (4). This implies that firm 3 will only change its behaviors depending on how the insider firms would depart from the premerger equilibrium. In turn, the merged entity must account for the strategic reaction of the outside firm when it sets prices and investments. In particular, the FOC for the optimal pricing decisions of the merged entity is

$$
\frac{\partial \pi_{12}}{\partial p_{i}}=q_{i}(p)+\left[p_{i}-\left(c_{i}-\mu_{i}\right)\right] \frac{\partial q_{i}(p)}{\partial p_{i}}+\left[p_{j}-\left(c_{i}-\mu_{j}\right)\right] \frac{\partial q_{j}(p)}{\partial p_{i}}=0
$$

where $i \neq j=1,2$.

The FOC for the optimal investment is the same as given in (6). Of note, because products are imperfect substitutes, the last term in 11 is positive, that is, $\frac{\partial q_{j}(p)}{\partial p_{i}}>0$. Thus, by comparing (11) and (5), ceteris paribus, the insiders have an incentive to raise their prices and decrease innovations. This is referred to as the coordination effect in the literature. Higher post-merger prices would reduce the merged entity's investment incentives. In other words, an insider would have to internalize the impacts of its price and investment on the partner's profit. Certainly, the behavior of the merged entity would account for the outsider's responses to the merger.

Now, we solve simultaneously the FOCs of the firms' profit maximizations. The equilibrium prices and investments post-merger are subscripted with " $M$ ". We can then compare the pre- and post-merger levels, and obtain: 
Proposition 2 Post-merger, the insiders' prices (respectively, investments) are higher (respectively, lower), whereas the outsider optimally sets a lower price, but higher investment, that is:

$\left\{\begin{array}{l}p_{i}^{M}>p_{i}^{E} \text { and } \mu_{i}^{M}<\mu_{i}^{E} \text { where } i=1,2 \\ p_{3}^{M}<p_{3}^{E} \text { and } \mu_{3}^{M}>\mu_{3}^{E}\end{array}\right.$

Proof. See the Appendix.

After the merger, the insiders will want to soften competition between them through adjusting prices and investments. More precisely, the merger would provide the symmetric firms an incentive to raise their prices to reduce negative effects on each other, as indicated in (11). Furthermore, the higher are the prices, ceteris paribus, the lower are the quantities. This leads to lower gains from investments and thereby reducing the insiders' incentives to invest in cost-reducing. In other words, the merger would result in higher prices, but lower investments of the insiders, that is, $p_{i}^{M}>p_{i}^{E}$ and $\mu_{i}^{M}<\mu_{i}^{E}$.

We can explicitly analyze the responses of the outsider who has a different cost structure. Post-merger, the insiders and the outsider set prices and investments differently. More precisely, firm 3 profitably reduces its price but increases investment regardless of whether it is relatively more or less efficient (i.e., the value of $a$ is positive or negative). Indeed, the insiders' higher prices provide the outsider an incentive to raise both the price and investment, as discussed previously. Proposition 2 indicates that the latter dominates the former effect, that is, $p_{3}^{M}<p_{3}^{E}$ and $\mu_{3}^{M}>\mu_{3}^{E}$.

Furthermore, the changes in the firms' prices and investments crucially depend on the degree of product substitutability and the cost asymmetry. More precisely, our comparative statics show that the lower level of the outsider's cost (that is, $\lambda$ is higher), the higher the changes in its price and investment (that is, $\frac{\partial\left(p_{3}^{E}-p_{3}^{M}\right)}{\partial \lambda}>0$ and $\left.\frac{\partial\left(\mu_{3}^{M}-\mu_{3}^{E}\right)}{\partial \lambda}>0\right)$. On the contrary, the insiders could only raise their prices slightly in this case (that is, $\frac{\partial\left(p_{i}^{M}-p_{i}^{E}\right)}{\partial \lambda}<0$ and $\frac{\partial\left(\mu_{i}^{E}-\mu_{i}^{M}\right)}{\partial \lambda}<0$ where $\left.i=1,2\right)$. Intuitively, if the asymmetry is high, the insiders are less able to raise the prices to avoid an important loss of the quantity, whereas the outsider has an incentive to significantly reduce its price. Similar phenomenons occur in the case of the high degree of substitutability, that is, a high value of $\gamma$. In short, the changes in the competitive behaviors of the outsider (insiders) after the merger are relatively more (less) significant when products are highly substitutable, and the outsider's cost is lower.

Next, we compute the firms' profits post-merger which we denote by $\pi_{i}^{M}$. The merger is profitable, that is, $\pi_{12}^{M}-2 \pi_{1}^{E}>0$, when the degree of substitutability is sufficiently low, as indicated in assumption $A 1$. Intuitively, when the products are close substitutes, an increase in the insiders' prices due to the coordination effect would imply a great loss of market share to the outsider. In other words, the merger could only be profitable when the decrease in the insiders' quantities is not significant, i.e., when the products are low substitutes. Put differently, when products are highly substitutable, the impacts of increased prices could not be compensated by the decreased quantities regarding the merged firms' profitability. It is straightforward to evaluate the merger impacts on the total investments and consumer surplus by comparing the pre- and post-merger levels. In particular, we let $T C^{M}$ and $C S^{M}$ denote respectively the total investment and consumer surplus post-merger. The merger effects on the investment and consumer surplus are represented by $\Delta T C=T C^{M}-T C^{E}$ and $\triangle C S=C S^{M}-C S^{E}$, respectively. Specifically, 
we can obtain post-merger total investments based on the firms' optimal investments and prices indicated in proposition 2 into (9). Then, we can rearrange $\Delta T C$ as a quadratic function of $\lambda$, as follows:

$$
\Delta T C=\digamma_{1} \lambda^{2}+\digamma_{2} \lambda+\digamma_{3}
$$

where $\digamma_{1}, \digamma_{2}, \digamma_{3}$ are higher degree polynomials of $c$ and $\gamma$, and $\digamma_{1}<0$. Thus, we can prove that $\Delta T C=0$ if $\lambda=\lambda_{1}$ or $\lambda=\lambda_{3}$, where the value of $\lambda_{1}$ is given in Assumption $A 3$, and $\lambda_{3}<\min \left(\lambda_{1}, \lambda_{2}\right)$. In other words, when $\lambda>\lambda_{3}$, the post-merger total investments are higher than the pre-merger level, that is, $\Delta T C>0$. Similarly, consumer surplus is improved due to the merger, that is, $\Delta C S>0$ if $\lambda>\lambda_{4}\left(>\lambda_{3}\right)$. We have thus established the following proposition.

Proposition 3 The merger spurs the total investments and consumer surplus if the outsider is sufficiently efficient compared to the insiders. In particular, we have:

$\left\{\begin{array}{l}\Delta T C>0 \text { if } \lambda>\lambda_{3} \\ \Delta C S>0 \text { if } \lambda>\lambda_{4}\end{array}\right.$

Proof. See the Appendix.

The intuitions behinds proposition 3 are quite clear. After the merger, the outsider and insider firms choose the opposite strategies. The net impacts depend on the changes in the firms' behaviors. In particular, if $\lambda$ is very large, that is, firm 3 has a very low exante marginal cost, then its large (ex-ante) quantity would raise the investment incentives. In this case, the insiders would only slightly decrease investments to avoid a great loss of market share, as previously discussed. Thus, we can show that when the outsider has a sufficiently low cost (i.e., $\lambda>\lambda_{4}$ ), both total investments and consumer surplus are higher after the merger. If $\lambda_{3}<\lambda<\lambda_{4}$, the total investments increase, whereas consumer surplus decreases. Interestingly, we observe that the value $\lambda_{3}$ could be negative for a sufficiently high level of $\gamma$ and a low value of $c$, that is when $c$ is near $\frac{1}{2}$ and $\gamma$ is near 0.478 . This result implies that in this market range, the total investments could be improved in the case of symmetric firms (see Appendix 2 for an illustration).

\section{Conclusion}

This paper attempts to analyze a simple merger case departing from the symmetric world. In particular, we investigate the firms' optimal prices and investments when products are differentiated. After the merger, the symmetric insiders raise prices and reduce investments to avoid negative effects on each other. Thus, this would give the outsider an incentive to increase investment and reduce its price because the profit derived from gaining from a larger market share is higher than that from setting a higher price. Thus, for a sufficiently large asymmetry, the effects of the outsider's aggressive strategy can overcompensate the negative impacts of the insiders' optimal choices. Although this paper could contribute to the existing literature to understand the competitive effects of horizontal mergers, it only explicitly examines the merger impacts in the case of triopoly in which the outsider firm can have a different marginal cost. Thus, it could be interesting to investigate the impacts of a merger between one of the high-cost firms and the low-cost one. This would provide a more comprehensive analysis and policy implications.

\footnotetext{
${ }^{5}$ This quadratic form reflects that $\lambda$ appears in both the firms' prices and quantities.
} 